\title{
GOTA SUSPENSA PARA AVALIAÇÃo DE ALDÉ́do TOTAL NO AR INTERNO E EXTERNO DO AMBIENTE
}

\author{
Elisabete A. Pereira e Arnaldo A. Cardoso
}

Departamento de Química Analítica, Instituto de Química, Universidade Estadual Paulista, Av. Prof. Francisco Degni s/n, 14800-900

Araraquara - SP

Parnendu K. Dasgupta

Department of Chemistry and Biochemistry, Texas Tech Universtiy, Lubbock, TX, 79409-1061, USA

Recebido em 4/1/00; aceito em 22/1/01

\begin{abstract}
SUSPENDED DROPLET FOR EVALUATION OF TOTAL ALDEHYDE IN OUTDOOR AND INDOOR AMBIENT. A sensitive and simple system was proposed for the in situ measurement of total aldehyde in outdoor or indoor ambient. The method is based on the use of a reagent drop as an useful interface to preconcentrate the sample prior to determination of total aldehyde as formaldehyde. The drop is formed at the tip of a cylindrical tube that contains two optical fibers placed on opposite sides and in contact with the reagent solution. One optical fiber carries the red light to the drop form a light emitting diode (LED). The transmitted light is measured by a second optical fiber/photodiode system. The analytical signal is read and converted into absorbance. The reagent solution of 3-methyl-2benzothiazoline hydrazone $(\mathrm{MBTH})$ forms a blue cation during reaction with formaldehyde that can be measured at $660 \mathrm{~nm}$. Some aspects of kinetics reaction formation of dye were reevaluated. The formaldehyde reacts with MBTH and forms the azine in about $12 \mathrm{~min}$. The oxidation of MBTH by Fe (III) and the formation of dye requires $3 \mathrm{~min}$. The absorbance of the reagent drop is proportional to the sampling time and to the analyte concentration. The absorbance signal increases with increased sample gas flow until a maximum is reached then decreases until it forms a plateau. The proposed method was evaluated using both outdoor and indoor samples, and it was shown to viable provide an accurate measure of total aldehyde.
\end{abstract}

Keywords: aldehyde; suspended droplet; air analysis.

\section{INTRODUÇÃO}

Depois dos hidrocarbonetos, os aldeídos são mais abundantes dos gases orgânicos presentes na atmosfera ${ }^{1}$. Estudos recentes têm mostrado que o formaldeído é a espécie mais abundante dos poluentes carbonílicos, seguido pelo acetaldeído ${ }^{1,2}$. Nos grandes centros urbanos brasileiros, como resultado do uso do álcool hidratado e gasolina misturada com álcool anidro como combustível de automóveis, o acetaldeído é geralmente mais abundante que o formaldeído na atmosfera ${ }^{3-5}$.

Várias são as fontes dos aldeídos presentes na atmosfera. A emissão direta ocorre como resultado da formação de aldeídos durante a combustão incompleta de combustível e biomassa ${ }^{6,7}$ e também resultado do seu largo uso como matéria prima. Os aldeídos também se formam diretamente na atmosfera como produto de reações de oxidações fotoquímicas de compostos orgânicos voláteis envolvendo óxidos de nitrogenio ${ }^{8-10}$. Durante episódios de smog, a formação de aldeído atmosférico pode chegar a ser dez vezes maior que a emitida diretamente ${ }^{10}$. Em regiões não urbanas, ricas em vegetação, a principal fonte de aldeído é proveniente de reações entre compostos orgânicos naturalmente emitidos pelas plantas e oxidantes presentes na atmosfera ${ }^{11,12}$.

Em ambientes fechados o formaldeído representa cerca de $60 \%$ do total dos aldeídos. Sua origem tem sido associada a complexos processos químicos e físicos que determinam geração, emissão e acúmulo do formaldeído ${ }^{12}$. As fontes usuais são carpetes, fumaça de cigarros e de frituras de alimentos, divisórias, madeiras compensadas, espumas isolantes, máquinas copiadoras ${ }^{13,14}$. Atualmente vem sendo considerada a sua formação, via reações químicas no ar como produto de reações entre compostos orgânicos voláteis (COVs) e oxidantes ${ }^{13,15}$.

Devido ao grande número de fontes e um tempo de residência na atmosfera de algumas horas ${ }^{16}$, o formaldeído, é um componente que tem sido encontrado na atmosfera de diferentes locais com uma ampla faixa de concentração. Em regiões urbanas sua concentração média varia de 20 a $75 \mathrm{ppb}^{9}$, já tendo sido relatados picos de $150 \mathrm{ppb}$ na cidade de Los Angeles ${ }^{17}$, enquanto que em regiões remotas sua concentração varia de 0,5 a $2 \mathrm{ppb}^{9,18}$. Níveis de até unidades de ppm têm sido encontrado em escritórios e residências ${ }^{19}$.

A toxicidade do formaldeído tem sido bastante estudada $a^{20,21}$. Ele é reconhecidamente irritante dos olhos e trato respiratório, mas nenhum estudo comprovou sua ação carcinogênica no homem sendo apenas descrito como altamente suspeito de ser um composto carcinogênico. Sua ação citotóxica e genotóxica tem sido atribuída a ataques nucleofílicos a grupos aminos e sulfidrilas presentes em muitas biomoléculas ${ }^{22}$.

A presença de aldeídos em concentrações elevadas em ambientes abertos é indicativa de atmosferas poluídas com COVs e espécies químicas envolvidas em reações fotoquímicas, enquanto em ambientes fechados sua presença pode ser indicativa de possíveis atmosferas insalubres que podem afetar atividades ocupacionais. Assim, é de fundamental importância que existam métodos sensíveis, com resultados rápidos e de fácil utilização para quantificação dos aldeídos de baixo peso molecular presente nos ambientes. O desenvolvimento de novos métodos e/ou a otimização dos já existente é de grande interesse tanto do ponto de vista analítico ambiental como toxicológico. Os principais métodos de análise de aldeídos são baseados em técnicas espectroscópicas e cromatografia ${ }^{23-25}$. Os métodos diretos que envolvem técnicas espectroscópicas na região do infravermelho requerem equipamentos com longos caminhos óticos e não portáteis. Já os métodos convencionais requerem etapas distintas de amostragem e determinação que além de consumir tempo do analista na manipulação da amostra podem ainda contribuir para ampliar os erros da análise. 
Nos últimos anos, a versatilidade no uso de pequenas gotas líquidas e filmes finos têm se mostrado como uma nova ferramenta para ser utilizada na coleta e determinação de analitos $\operatorname{gasosos}^{26-31}$. Suas principais vantagens são a amostragem do analito e posterior determinação sem manipulação da amostra, aliada a boa sensibilidade e baixo tempo de resposta analítica. No trabalho aqui descrito, foram reavaliadas as condições da reação entre o formaldeído e o reagente 3- metil-2benzoatiazona hidrazona (MBTH) e propostas as melhores condições para análise de aldeídos de baixo peso molecular utilizando a técnica da gota suspensa. O método baseia-se no uso de uma gota de solução reagente MBTH como interface para amostrar e determinar aldeídos gasosos. As medidas de absorbância são feitas diretamente na gota logo depois de finalizada a amostragem e permitem a determinação dos aldeídos de baixo peso molecular com resultados de análise próximos do tempo real.

\section{MATERIAIS E MÉTODOS}

\section{Reagentes}

Todos os reagentes foram preparados e estocados de acordo com o procedimento para determinação do conteúdo de formaldeído na atmosfera pelo método do $\mathrm{MBTH}$, descrito em manual de amostragem e análise de $\mathrm{ar}^{32}$. A solução de MBTH (Aldrich) usada na coleta e determinação foi de $2,32 \times 10^{-3} \mathrm{~mol} \mathrm{~L}^{-1}$. A solução oxidante continha $1,0 \mathrm{~g}$ de ácido sulfâmico e $0,63 \mathrm{~g}$ de cloreto de ferro (III) diluído até $100 \mathrm{~mL}$ com água deionizada.

\section{Geração da Mistura Gasosa Padrão}

$\mathrm{O}$ esquema do arranjo montado para gerar a mistura gasosa padrão de formaldeído usado nestes estudos está mostrado na Figura 1. $\mathrm{O}$ ar foi filtrado por um conjunto de colunas (A), contendo em cada uma delas, solução sulfocrômica depositada sobre sílica gel, carvão ativo,sílica gel e peneira molecular. Parte do ar purificado (VF1) passa pela câmara (C) onde foi colocado um tubo de permeação com taxa de emissão de $30 \pm 2 \mathrm{ng} \mathrm{m^{-1 }}$ de formaldeído $\left(25,0 \pm 0,1^{\circ} \mathrm{C}\right)$. A mistura gasosa gerada pode ser convenientemente diluída pelo restante do ar purificado (VF2). A vazão do gás padrão utilizado nos testes analíticos pode ser controlada pela válvula (VF3), que descarta o excesso do gás padrão gerado.

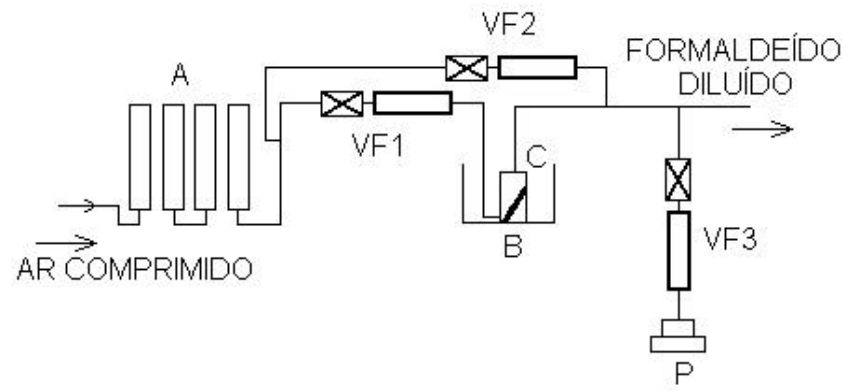

Figura 1. Esquema do sistema para geração da atmosfera padrão de formaldeído. A: Conjunto de colunas contendo: peneira molecular, sílica /sulfocrômica, peneira molecular / sílica e carvão ativo / sílica gel; VF: conjunto de válvula de agulha e controlador de fluxo; $B$ : Banho termostático; C: Câmara de amostragem com tubo de permeação; P: Bomba de Vácuo

\section{Sistema de Medidas}

O sensor foi construído com um tubo de plástico com $6 \mathrm{~mm}$ de diâmetro interno fixado na posição vertical, para funcionar como um gerador de gotas (Figura 2). Um tubo de Teflon (diâmetro interno $0,8 \mathrm{~mm}$ ) conectado ao gerador de gota permite introduzir com uma microseringa, as soluções para formar a gota. $\mathrm{Na}$ extremidade inferior do tubo, fixou-se duas fibras ópticas posicionadas uma de frente para a outra e com suas outras extremidades, uma ligada a uma fonte de luz proveniente de um LED (light emitting diode) vermelho com $\lambda_{\max } 660 \mathrm{~nm}$ e a outra extremidade ligada a um fotodiodo para receber a luz que atravessa a gota formada. Foi conectado também um fotodiodo junto ao LED para gerar uma fotocorrente de referência. Os fotodiodos foram acoplados a um sistema para leitura e transformação das fotocorrentes em sinal de absorbância ${ }^{33}$. A fotocorrente gerada no detetor não é somente a luz transmitida pela gota, mas também a resultante de espalhamentos de luz proveniente de processos reflexão e refração que ocorrem no interior da gota, por esta razão o sinal é lido como absorbância aparente.

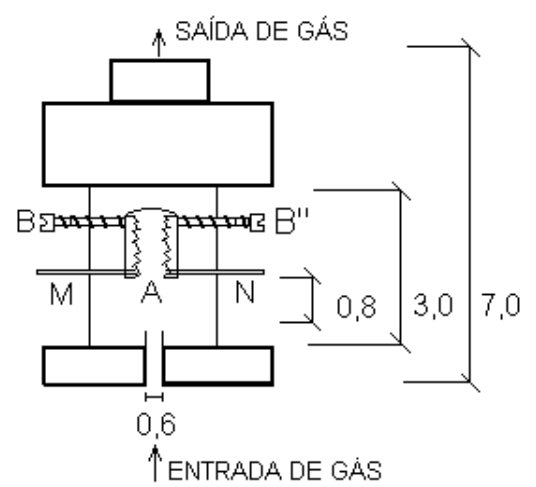

Figura 2. Esquema do sensor formado pelo gerador de gotas e a câmara de amostragem, dimensões estão em centímetros. A: gerador de gotas; $B$ e B": parafusos; $M$ e $N$ : fibras óticas.

O sensor foi fixado dentro de um tubo plástico que servia como câmara de amostragem. O gás de amostragem era introduzido na câmara de amostragem por um tubo situado na parte inferior, fixado a uma distância de $0,8 \mathrm{~cm}$ do gerador de gotas.

\section{RESULTADOS E DISCUSSÃO}

\section{Considerações Iniciais}

Os aldeídos reagem com a solução de MBTH para formar um cátion azul. A reação ocorre em três etapas: (1) o aldeído reage com o MBTH para formar azina correspondente, (2) ferro (III) adicionado a solução oxida MBTH para formar um cátion reativo e (3) a azina e o cátion reativo se combinam para formar um cátion azul que possui absorbância máxima em 628 nm (Figura 3).

A reação não é imediata, o tempo recomendado pelos autores do método original ${ }^{34,35}$, é de uma hora para a primeira etapa da reação e mais 12 minutos após a adição de $\mathrm{Fe}$ (III) para proceder a leitura do sinal de absorbância. A reação não é específica. Várias classes de compostos reagem como MBTH produzindo compostos coloridos: aminas aromáticas, bases de Schiff e iminos heterociclícos, mas em amostragens de ar estas espécies não estão presentes ou não são solúveis em água. Estes fatos garantem uma certa especificidade das análises de aldeídos no ar. Já a absorbância dos aldeídos alifáticos diminui com o aumento da cadeia ${ }^{32}$. Para soluções de mesma concentração, acetaldeído e propionaldeído respectivamente produzem uma absorbância de $80 \%$ e $58 \%$ da absorbância do formaldeído. O resultado portanto representa o aldeído total que pode ser expresso como formaldeído. 
<smiles>C=N/N=C1\Sc2cc3ccc2N(C)C1=NN=C1Sc2ccccc2N1C3=NN=c1[nH]c2ccccc2s1</smiles>

(B)

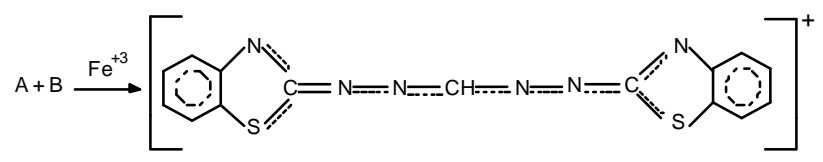

(C)

Figura 3. Reação de formação do corante cátion azul. (A): reação entre MBTH e aldeído com formação da azina; $(B)$ : reação de oxidação do MBTH com $\mathrm{Fe}(\mathrm{III})$ em meio ácido; $(C)$ : reação entre os produtos das etapas $(A)$ e (B) para formação do corante cátion azul $(C)$.

\section{Estudo Sobre o Comportamento da Reação Entre o MBTH e o Formaldeído}

Para a determinação colorimétrica de aldeídos do ar tem sido recomendado o uso de MBTH como reagente de formação de corante ${ }^{32}$. Mas, apesar do largo uso desta reação, poucos são os estudos sobre o comportamento cinético da reação. As informações existentes na literatura praticamente se restringem aquelas feitas quando o método foi proposto ${ }^{32}$. Como o tempo sugerido para que ocorra a formação do corante é relativamente longo, procurou-se reavaliar esta transformação em função do tempo. Iniciou-se o estudo pela formação da azina que é descrita como a etapa mais lenta do processo. Para acompanhar a primeira etapa da reação foram traçados os espectros das soluções das espécies envolvidas utilizando-se um espectrofotômetro (Hitachi U 2000). O espectro de absorção da solução de MBTH apresenta 3 picos de absorção, como mostra a Figura 4. Já a solução de formaldeído não absorve nesta faixa de comprimento de onda. A solução resultante da reação entre o MBTH e o formaldeído lida uma hora após a mistura, apresenta uma absorção característica acima de $290 \mathrm{~nm}$, com uma banda larga de absorção que chega até próximo de $320 \mathrm{~nm}$, que caracteriza a azina formada como mostra a Figura 5.

\section{Influência da temperatura na velocidade da reação da formação da azida}

O efeito da temperatura no desenvolvimento da reação entre formaldeído e MBTH foi determinada misturando-se $10,0 \mathrm{~mL}$ de solução de MBTH $\left(2,32 \times 10^{-6} \mathrm{~mol}\right) \operatorname{com} 1,0$ $\mathrm{mL}$ formaldeído $\left(1,33 \times 10^{-6} \mathrm{~mol}\right)$ em diferentes temperaturas. Alíquotas da solução resultante foram retiradas a cada 10 minutos e medidas em espectrofotômetro no comprimento de onda de $\lambda=307 \mathrm{~nm}$. Os resultados apresentados na Figura 6 mostraram que a absorbância do produto da reação, aumenta rapidamente até atingir um patamar e que este produto é favorecido pelo aumento da temperatura até $31,9^{\circ} \mathrm{C}$. Passados 10 minutos do início da reação, cerca de $75 \%$ do produto já foi formado à temperatura de $24,0^{\circ} \mathrm{C}$ e cerca $93 \%$ para temperatura de $31,9^{\circ} \mathrm{C}$. Observa-se que para $43^{\circ} \mathrm{C}$ a quantidade do produto formado diminui, fato que pode estar relacionado ao escape de formaldeído do meio reacional, favorecido quando temperaturas superiores a $35^{\circ} \mathrm{C}$ são utilizadas ${ }^{36}$.

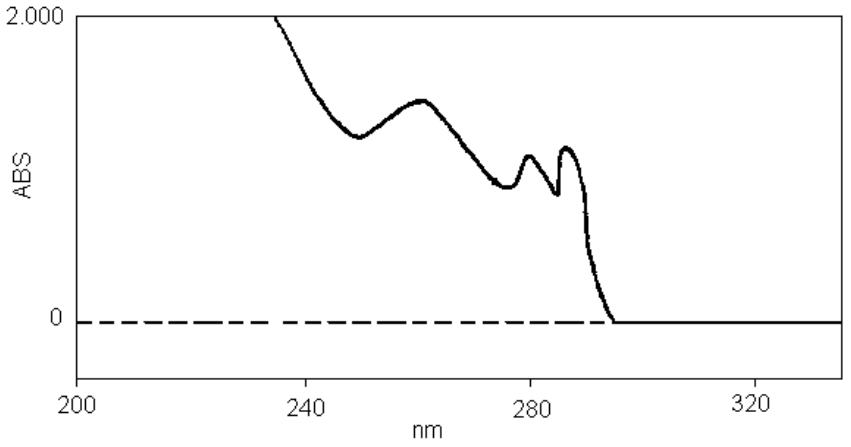

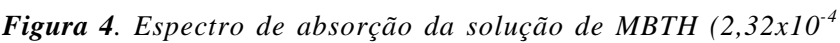
mol/L). Máximos de absorção : $256 \mathrm{~nm}, 280 \mathrm{~nm}$ e $286 \mathrm{~nm}$.

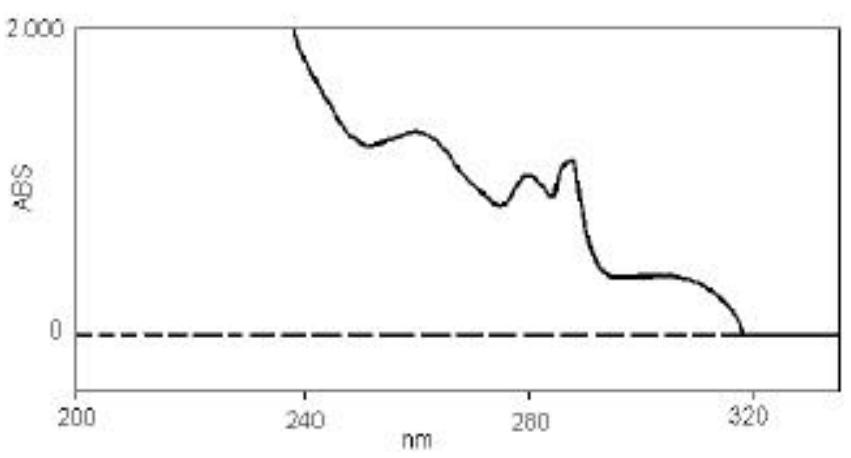

Figura 5. Espectro de absorção da mistura entre $10,0 \mathrm{~mL}$ de MBTH $\left(2,32 \times 10^{-6} \mathrm{~mol} / \mathrm{L}\right)$ e $1,0 \mathrm{~mL}$ de $\mathrm{CH}_{2} \mathrm{O}\left(1,33 \times 10^{-6} \mathrm{~mol} / \mathrm{L}\right) .256 \mathrm{~nm}, 280 \mathrm{~nm}$, $286 \mathrm{~nm}$ e $307 \mathrm{~nm}$.

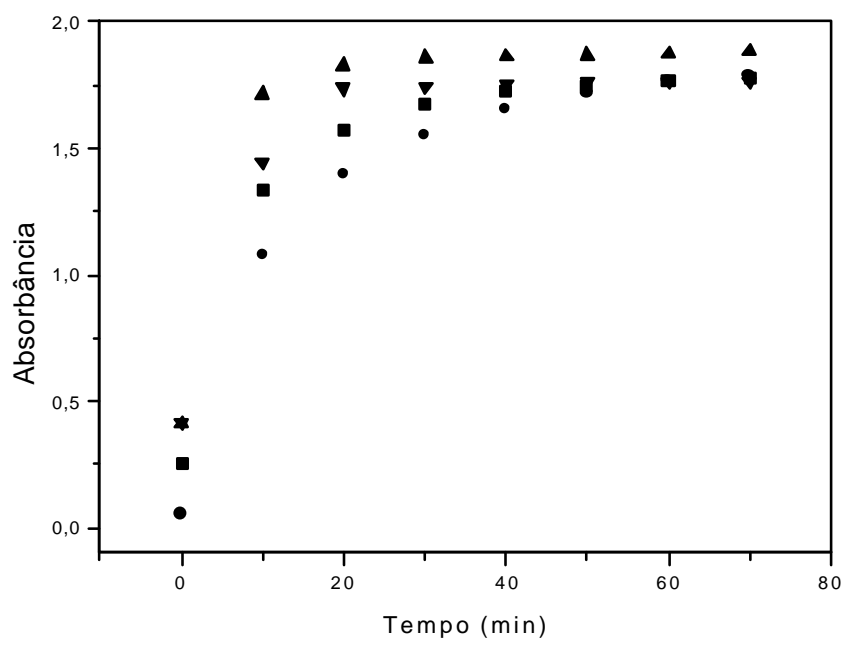

Figura 6. Estudo da influência da temperatura sobre a formação da azina. Comprimento de onda selecionado $\lambda=307 \mathrm{~nm}$. Legenda: $\mathbf{\square} 15,1^{\circ} \mathrm{C}$; $\Delta 31,9^{\circ} \mathrm{C} ; \boldsymbol{\bullet} 24,0^{\circ} \mathrm{C} ; \boldsymbol{\nabla} 43,0^{\circ} \mathrm{C}$.

\section{Influência do tempo na formação do corante}

Foi também avaliado o tempo necessário para formação do produto colorido a partir da adição da solução de $\mathrm{Fe}(\mathrm{III})$. Inicialmente foram misturados $10,0 \mathrm{~mL}$ de solução de MBTH $(2,32 \mathrm{x}$ $\left.10^{-6} \mathrm{~mol}\right)$ com $1,0 \mathrm{~mL}$ de solução formaldeído $\left(1,30 \times 10^{-6} \mathrm{~mol}\right)$. Alíquotas de $10 \mathrm{~mL}$ foram retiradas imediatamente após o tempo de 2, 5, 10 e 60 minutos, sendo adicionadas a cada uma dessas alíquotas $2,0 \mathrm{~mL}$ da solução oxidante $\mathrm{Fe}(\mathrm{III})$ para a formação do corante. A variação da absorbância $(\lambda=628 \mathrm{~nm})$ da solução resultante foi acompanhada ao longo de 12 minutos no 
espectrofotômetro (Figura 7). Os resultados do experimento mostraram que a presença do oxidante no meio reacional bloqueia a reação entre MBTH e formaldeído e somente a azina já formada é transformada em corante. Não ocorre variação significativa do sinal da absorbância para tempos acima de 10 minutos de reação entre formaldeído e MBTH, o que confirma o experimento anterior que sugere que a maior parte da azina se forma neste tempo de reação. Mostrou também que o desenvolvimento da cor aumenta rapidamente até chegar a um patamar, cerca de 3 minutos após a adição do oxidante.

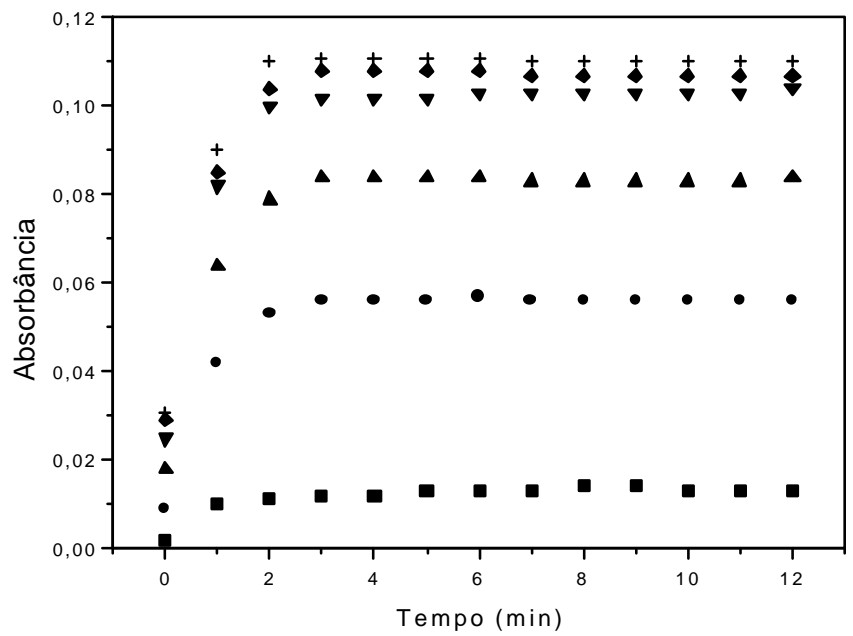

Figura 7. Estudo da influência do tempo de reação entre $\mathrm{MBTH}$ e $\mathrm{CH}_{2} \mathrm{O}$ na absorbância do produto final (cátion azul). $O$ oxidante foi adicionado após diferentes tempos de reação entre $\mathrm{MBTH}$ e $\mathrm{CH}_{2} \mathrm{O}$. Legenda:

- 0 min; $2 \mathrm{~min} ; \boldsymbol{\Delta} 5 \mathrm{~min} ; \boldsymbol{\nabla} 10 \mathrm{~min} ; \bullet 20 \mathrm{~min} ;+60 \mathrm{~min}$.

Os experimentos indicam que pode-se diminuir o tempo total de reação de formação do corante para cerca de 15 minutos, 12 13 minutos de espera entre a reação de MBTH e formaldeído e mais 3 minutos após a adição da solução oxidante de (Fe III). Possivelmente estudos anteriores não deram devida atenção ao aspecto tempo porque, ele era pouco significativo. $\mathrm{O}$ método descrito para formaldeído usando o reagente MBTH sugere amostragens de mais que 8 horas usando soluções de MBTH em borbulhadores. Neste caso pouco significado tem a espera de mais uma hora para determinação do produto. Mas, na proposta deste trabalho, onde a amostragem é feita em poucos minutos, esse tempo passa a ser significativo. É necessário ainda salientar que nos experimentos acima, todo aldeído foi colocado para reagir no instante inicial da reação, mas quando são feitas amostragens utilizando soluções de MBTH para coletar o aldeído, ele vai sendo absorvido durante toda a amostragem, assim o tempo de reação é diferente para cada molécula amostrada. Portanto em condições usuais de amostragem utilizando solução de MBTH como solução absorvente de aldeídos, a formação da azina, dez minutos após finalizada a amostragem, já deve ser maior que a calculada aqui.

Experimentos complementares mostraram que outros parâmetros que poderiam influenciar a reação, possuíam o comportamento sugerido por trabalhos da literatura ${ }^{35}$ : a concentração da solução de MBTH não afeta a absorbância final mas deve estar em excesso em relação ao aldeído; a maior absorbância foi obtida quando se trabalhou com uma razão molar de $1 \mathrm{MBTH}: 2 \mathrm{Fe}(\mathrm{III})$; o uso de água oxigenada como oxidante não apresentou os resultados esperados.

\section{Avaliação do Sensor Acoplado a Gota}

\section{Absorbância em função do volume da gota}

O volume da gota é uma variável significativa em medidas de absorbância. O volume da gota está correlacionado com o caminho ótico e com reflexões de luz pela superfície côncava interna da gota que pode atuar como um colimador de luz $z^{37}$. Para avaliar como esta variável influencia as medidas de absorbância, utilizou-se uma solução de MBTH 0,05\%. Para obter um gotejamento constante, um frasco contendo esta solução foi colocada cerca de 90 centímetros acima do gerador de gota. A solução foi levada até o gerador de gota através de um fino tubo de Teflon (diâmetro interno $0,8 \mathrm{~mm}$ ) pela ação da gravidade, formando um gotejamento constante. A absorbância foi registrada durante um intervalo de tempo, como mostra a Figura 8. Como neste intervalo de tempo a frequiência de gotejamento foi constante, podemos observar a dependência da absorbância com o volume da gota. Em um experimento paralelo determinou-se que o volume máximo de cada gota é $170 \mu \mathrm{L}$. Pode-se portanto concluir que existe um intervalo de volume de gotas entre $110 \mu \mathrm{L}$ e $150 \mu \mathrm{L}$ onde a variação do valor de absorbância não é significativa.

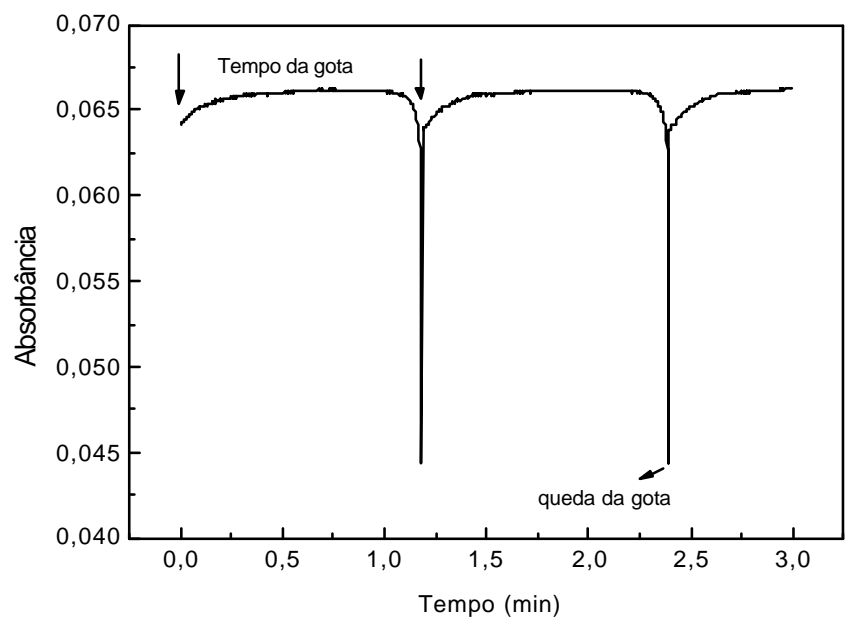

Figura 8. Absorbância como função do ciclo da gota com solução MBTH 0,05\%.

\section{Influência do tempo e vazão de amostragem}

Para se estabelecer o procedimento de amostragem dois parâmetros precisaram ser avaliados, o tempo e a vazão de amostragem. Com a vazão de amostragem de $110 \mathrm{~mL} / \mathrm{mim}$ e concentração de $61 \mathrm{ppb}$ de formaldeído foram feitas amostragens com intervalo de tempo variando de 1 a 7 minutos, seguindo com posterior determinação do formaldeído. A Figura 9 mostra que o sistema responde de forma linear à variação da quantidade de formaldeído que chega à gota durante os diferentes tempos de amostragens estudados, sem a aparente saturação da gota quanto a capacidade de coleta e reação. Tendo em vista estes resultados selecionou-se o tempo de amostragem de 3 minutos por ser intermediário entre eles. Mas caso seja necessário melhorar o limite de quantificação, o melhor procedimento será aumentar o tempo de amostragem sem prejuízo para o método. Indiretamente, o experimento mostra que a evaporação de parte da gota, mesmo com umidade relativa muito baixa da mistura gasosa padrão, não é significativa para afetar os resultados, fato que confirma resultados anteriores que avaliaram o efeito da umidade relativa do ar em experimentos semelhantes ${ }^{26,28}$.

A vazão de amostragem foi estudada para o intervalo de 110 a $541 \mathrm{~mL} / \mathrm{min}$ com a concentração de formaldeído mantida em 61 ppbv e tempo de amostragem de 3 minutos. Os resultados (Figura 10) mostraram que o sinal analítico aumenta linearmente com o aumento da vazão de amostragem até $210 \mathrm{~mL} /$ min, acima desta vazão, o sinal analítico decresce e tende a 
formar um patamar. Como o patamar significa saturação da gota quanto a capacidade de amostrar e determinar o formaldeído, é conveniente trabalhar com vazões menores que $210 \mathrm{~mL} / \mathrm{min}$.

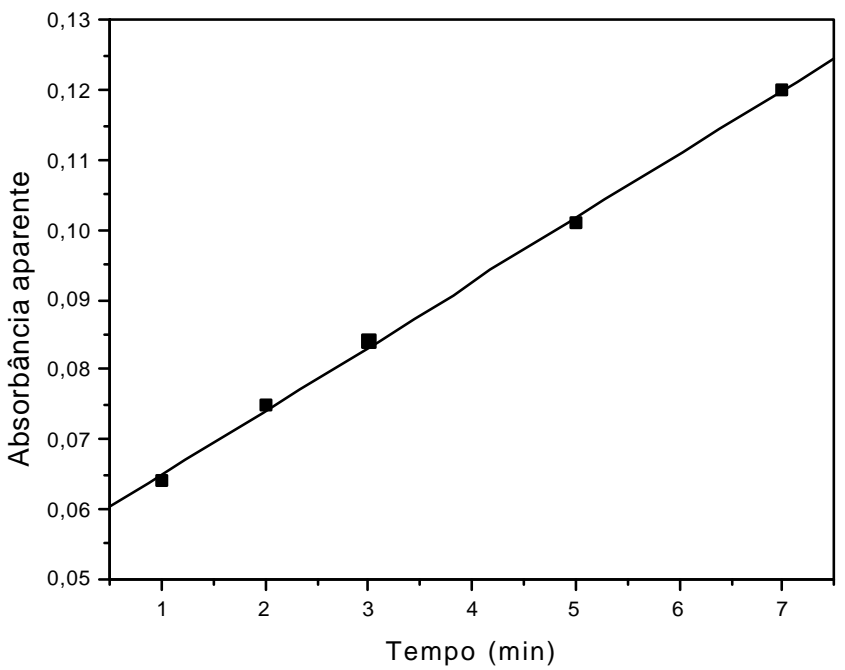

Figura 9. Comportamento do sistema de análise frente a diferentes tempos de amostragens. Concentração de $\mathrm{CH}_{2} \mathrm{O} 61 \mathrm{ppb}$, vazão de amostragem $110 \mathrm{~mL} / \mathrm{min}$.

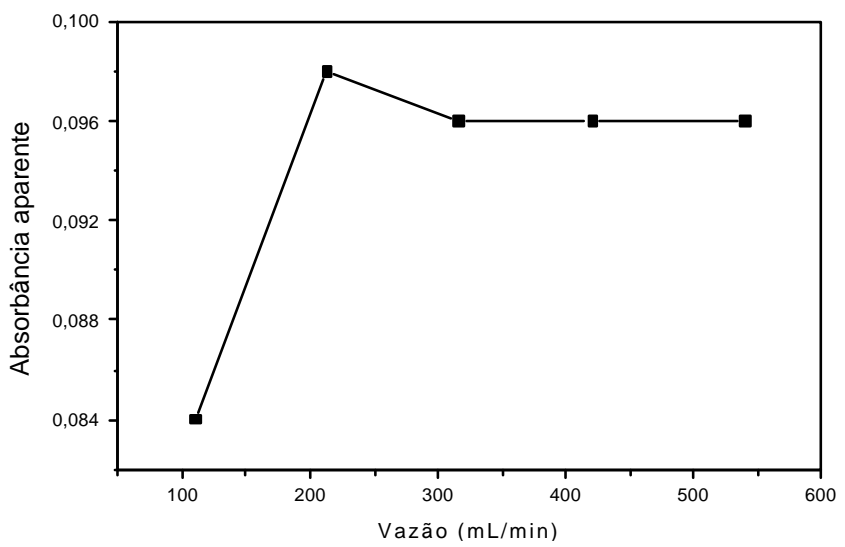

Figura 10. Comportamento do sistema frente a diferentes vazões de amostragem. Concentração de formaldeído $61 \mathrm{ppb}$, tempo de amostragem 3 minutos.

Com os resultados obtidos nestes experimentos foi possível propor o procedimento de análise:

a. formação da gota de amostragem: com auxílio de uma microseringa foi introduzido $110 \mathrm{~mL}$ da solução de $\mathrm{MBTH}$ para formação da gota;

b. amostragem de formaldeído por aspiração do ar para dentro da câmara de amostragem durante 3 minutos com uma vazão de $150 \mathrm{~mL} / \mathrm{min}$;

c. finalizada a amostragem, a gota permaneceu em repouso por cerca de 12 minutos;

d. adicionou-se com uma microseringa $40 \mathrm{~mL}$ da solução oxidante e registra-se o sinal de absorbancia por 3 minutos;

f. dispensa-se a gota com adição de solução de MBTH e formação de nova gota, possibilitando que novo ciclo de amostragem seja feito.

\section{Curva de Calibração}

A curva de calibração foi traçada utilizando-se amostras de ar concentração de formaldeído variando de 27 a 127 ppb (32 a $151 \mu \mathrm{g} / \mathrm{m}^{3}$ ). Não se testou valores acima desta concentração, devido à falta de interesse ambiental e valores menores não foram possíveis de serem obtidos devido a limitações experimentais. O tubo de permeação possuía uma taxa de emissão de formaldeído que exigia grandes volumes de ar limpo para se obter concentrações menores que $27 \mathrm{ppb}$. A dificuldade de se trabalhar em escala de laboratório com grandes vazões é que os erros associados ao controle e medida destas vazões limitam a obtenção de padrões muito diluídos de gases, como já foi discutido por Mac Taggart e colaboradores ${ }^{38}$. O sinal da absorbância para a faixa avaliada, mostrou-se linear para este intervalo de concentração $(\mathrm{r}=0,9949 \mathrm{sd}=0,000492)$. A reta pode ser representada pela equação:

$$
\mathrm{A}=0,00892+0.00109[\mathrm{HCOH}]
$$

onde A é a absorbância e [HCOH] concentração do formaldeído em ppb. O limite de detecção baseado no critério de três vezes o desvio padrão do branco é de 5 ppb.

\section{Avaliação do Método Com Amostras Reais}

O local escolhido para testar a metodologia, foi o Instituto de Química de Araraquara (SP) pelas facilidades de trabalho. Foram feitas análises pontuais em algumas áreas externas e internas do Instituto, avaliou-se também a concentração de aldeído ao longo do dia. As Tabelas 1 e 2 apresentam alguns dos valores encontrados para aldeídos e calculados como formaldeído total.

Tabela 1. Análise de aldeído total utilizando o sistema de gotas suspensas. Tempo de amostragem de 3 minutos e vazão de amostragem de $150 \mathrm{~mL} / \mathrm{min}$.

\begin{tabular}{lcc}
\hline Local & Horário & $\begin{array}{c}\text { Conc. de } \\
\mathrm{CH}_{2} \mathrm{O}(\mathrm{ppb})\end{array}$ \\
\hline Laboratório de Analítica & $10: 30$ & 84,0 \\
Jardim entre os laboratórios & $11: 20$ & 72,0 \\
da Analítica e Orgânica & & \\
Entrada do Instituto & $15: 30$ & 50,0 \\
Biblioteca & $20: 10$ & 23,0 \\
\hline
\end{tabular}

Tabela 2. Amostragem e análise de aldeído total ao longo do dia, utilizando sistema de gotas suspensas. Tempo de amostragem de $3 \mathrm{~min}$, vazão de amostragem de $150 \mathrm{~mL} / \mathrm{min}$.

\begin{tabular}{cccc}
\hline Horário & Temp. ${ }^{\circ} \mathrm{C}$ & $\begin{array}{c}\text { umidade } \\
\text { do } \text { ar\% }\end{array}$ & $\begin{array}{c}\text { Conc. } \\
\mathrm{CH}_{2} \mathrm{O}(\mathrm{ppb})\end{array}$ \\
\hline 9,00 & 21,6 & 61,0 & 23,0 \\
$10: 10$ & 23,5 & 59,0 & 24,0 \\
$11: 30$ & 27,0 & 51,0 & 62,5 \\
$12: 45$ & 28,1 & 50,7 & 69,8 \\
$13: 45$ & 28,6 & 49,2 & 91,8 \\
$14: 50$ & 27,8 & 47,4 & 72,5 \\
$16: 00$ & 27,4 & 46,6 & 53,3 \\
$17: 30$ & 26,6 & 50,0 & 40,4 \\
\hline
\end{tabular}

Os resultados encontrados para análises dos laboratórios mostraram valores relativos elevados certamente provenientes de fontes de emissões internas. Análises feitas 50 minutos após no jardim entre os laboratórios de pesquisa da Química Analítica e Química Orgânica (ambiente semi aberto) mostraram valores $15 \%$ menores. Já as análises feitas no período da tarde, na portaria do IQ, portanto longe da influência dos laboratórios, mostraram valores menores ainda, confirmando a presença de uma fonte pontual e localizada. A biblioteca apresentou valores 
relativos baixos, o que sugere que a emissão interna deve ser relativamente pequena. Já as análises feitas ao longo do dia em ambiente aberto e longe da influência dos laboratórios mostraram uma forte correlação entre a concentração de aldeído no ar e a insolação. $\mathrm{O}$ valor do aldeído chegou a alcançar quatro vezes o valor do início da manhã e posteriormente caiu ao longo da tarde. A variação da concentração do aldeído ao longo do dia, é um comportamento esperado, confirmando os trabalhos clássicos da literatura ${ }^{9}$. Ainda deve se salientar que estes últimos resultados foram obtidos em condições propícias para formação de aldeído, dia ensolarado, seco, pouco vento e com uma névoa de fumaça resultante da queima intensa de grandes áreas da lavoura de cana de açúcar, que estava em plena safra.

\section{CONCLUSÕES}

O método desenvolvido para determinação de aldeídos de baixo peso molecular mostrou-se conveniente para fazer acompanhamento da variação de aldeídos tanto em ambientes externos como internos. A facilidade e a capacidade de analisar amostras de ar em um período bastante curto, de cerca de 18 minutos entre cada análise presta-se muito bem para estudos sobre a variação destes aldeídos ao longo do tempo, com os valores de concentração dos aldeídos sendo conhecidos em um tempo muito próximo do real. Medidas que permitiriam fazer se inferências sobre a presença de compostos orgânicos voláteis no ar, oxidantes e atividade fotoquímica. Já em ambientes internos, estas medidas seriam suficientes para detectar possíveis fontes e avaliar a qualidade do ar ambiente.

O método desenvolvido é de fácil utilização e foi construído com materiais de baixo custo. A adaptação de um microcomputador portátil permitiria uma maior mobilidade do equipamento facilitando a operação em monitoramento ambiental.

\section{AGRADECIMENTOS}

E.A.P. agradece a FAPESP pela bolsa de mestrado concedida para desenvolver este trabalho. Pesquisa financiada por FAPESP/CNPq.

\section{REFERÊNCIAS}

1. Graedel, T. E.; Atmospheric Chemical Compounds: Sources, Occurrence and Biossay, Ed.;,Academic Press, Orlando, 1986; p 295.

2. Grosjean, D.; Environ.Sci.Technol. 1982,16, 254.

3. Tanner, R. J.; Miguel A. H.; Andrade, J. B.; Gaffney, J. S.; Streit, G. E.; Environ Sci. Technol. 1988, 22, 1026

4. Grosjean, D.; Miguel, A. H.; Tavares, T. M.; Atmos. Environ. 1990, 24, 101.

5. Brickus, L. S. R.; Cardoso; J. N.; Aquino Neto, F. R.; Environ Sci. Technol. 1998, 32, 3485.

6. Baugh, J.; Ray, W.; Black ,F.;. Atmos. Environ. 1987, 21, 2077.

7. Carlier, P.; Hannachi, H.; Mouvier, G.; Atmos. Environ. 1985, 19, 623.

8. Betterton, E. A.; In Gaseous Pollutants, Characterization and Cycling, Ed.; J. O. Nriagu, John Wiley \& Sons Inc., New York, 1992; p 335.

9. Finlayson-Pitts, B. J.; Pitts Jr. Atmospheric Chemistry:
Fundamentals and Experimentals Techniques; Ed.; John Wiley \& Sons; New York, 1986.

10. Grosjean ,D., Environ. Sci. Technol. 1991, 25, 710.

11. Lloyd C. A.; Atkinson R.; Lurmann F. W.; Nitta, B.; Atmos. Environ. 1983, 17, 1931.

12. Shepson, P. B.; Shepson; D.; Hastie ,R., Schiff, H. I.; Polizzi, M.; Bottenheim, J. W.; Anlauf, K.; Mackay, G. I.;. Karecki, D. R.; Atmos. Environ. 1991, 25, 2001.

13. Zhang, J.; He, Q.; Lloy, P. J.; Environ Sci. Technol. 1994, $28,146$.

14. Kelly, T. J.; Smith, D. L.; Satola, J.; Environ Sci. Technol. 1999, 33, 81 .

15. Wecheler, J. C.; Hodgson, A. T.; Wooley, J. D.; Environ. Sci. Technol. 1992, 26, 2371.

16. Moortgat, G. K.; Slemr, F.; Seiler, W.; Warneck, P.; Chem. Phys. Lett. 1978, 54, 444.

17. Altshuller, A. P.; McPherson, S. P.; J. Air Pollut. Contrl. Assoc. 1963, 13, 109.

18. Carlier, P.; Hannachi, H. e Mouvier, G.; Atmos. Environ. 1986,20, 2079.

19. Meyer, B.; Hermanns, K.; J.Environ.Health. 1985, 48,57.

20. Hileman, B.; Environ.Sci. Technol. 1984, 18, 216 A.

21. Matthews,T. G.; Reed,T. J.; Tromberg,B. J.; Daffron, C. R.; Hawthorne, A. R.; In Advances In Chemistry Serie 210 Formaldehyde - Analytical Chemistry and Toxicology, Ed.; Twroski, American Chemical Society, Washington, DC, 1985; p 131.

22. Wilson, V. L.; Foiles, P. G.; Chung, F. L.; Povey, A. C.; Frank, A. A.; Harris, C. C.; Carcinogenesis. 1991, 12, 1483.

23. Pickard, A. D.; Clark, E. R.; Talanta. 1984, 31, 763.

24. Vairavamurthy, A.; Roberts, J. M.; Newman, L.; Atmos. Environ. 1992, 26, 1965.

25. Cardoso, A. A.; Pereira, E. A.; Anais Assoc. Bras. Quim. 1999, 48, 63.

26. Liu, S.; Dasgupta, P. K.; Anal. Chem. 1995, 67, 2042.

27. Cardoso, A. A.; Dasgupta, P. K.; Anal. Chem. 1995, 67, 2562.

28. Cardoso, A. A.; Liu, H.; Dasgupta, P. K.; Talanta. 1997, 44, 1099.

29. Pereira, E. A.; Dasgupta, P. K.; Intern. J. Environ. Anal. Chem. 1997, 66, 201

30. Milani, M. R.; Gomes Neto, J. A.; Cardoso, A. A.; Microchem. J. 1999, 62, 273

31. Pretto, A.; Milani, M. R.; Cardoso, A. A.; J. Environ. Monit. 2000, 2, 566

32. Bryan, R. J.; Hyde, D. L.; Levaggi, D. A.; Locke, D. C.; Rasmussen, R. A.; Warner, P. O; In Method for Air Sampling and Analysis, 3 rd, Ed.; Lodge, J. P.; Lewis, Chelsea, New York, 1989; p 279.

33. Liu, H.; Dasgupta, P. K.; Talanta. 1993,40,1331.

34. Sawicki, G; Hauser, T. R.; Stanley, T. W.; Elbert, W.; Anal. Chem. 1961, 33, 93.

35. Hauser, J. R.; Cummins, R. L.; Anal. Chem. 1964, 36, 679.

36. Kirk, R.; Othmer, D.; Encyclopedia of Chemical Technology, Ed. The Interscience Encyclopedia, INC. New York, 1967, V. $6, \mathrm{p} 857$.

37. McMillan, N. D.; O’Mongain, E.; Walsh, J.; Breen, L.; Power, M. J.; O’Dea, J. P.; Kinsella, S. M.; Delly, M. P.; Hammil, C.; Orr, D.; Optical Engineering. 1994, 33, 3871.

38. Mactaggart, D. L.; Kagel, R. A.; Farwell, S.; JAPCA. 1987, 37, 143. 